\title{
Calcium/Calmodulin-dependent Serine Protein Kinase-associated Neuro-gastrointestinal Disorder: First Report of a Patient With Hirschsprung's Disease
}

\section{Introduction}

Although Hirschsprung's disease (HD) is the most common congenital malformation of the enteric nervous system in children, its genetic etiology remains unclear. ${ }^{1}$ Calcium/calmodulin-dependent serine protein kinase (CASK) is associated with digestive diseases, including cancer and proctitis; ${ }^{2,3}$ however, CASK mutations have not been shown to cause HD. We report a case involving a male child with CASK mutation and $\mathrm{HD}$, demonstrating the disease's typical neurological manifestations.

\section{Case Report}

We assessed the second child born at term through vaginal delivery to a healthy non-consanguineous Chinese couple. Twentytwo days after birth, he developed refractory constipation with abdominal distension. At 3 months, he had no social smile, could not control his head, and presented with refractory seizures. The head circumference was $38 \mathrm{~cm}$ (-2 SD). Generalized hypotonia with normal tendon reflex and abdominal distension showing a frog belly shape were observed. Abdominal radiography indicated congenital megacolon (Fig. 1A and 1B), and electroencephalogram results revealed hypsarrhythmia and epileptic spasms in clusters (Fig. 1C). Brain MRI revealed pontocerebellar hypoplasia (PCH) (Fig. 2A). Routine biochemical and metabolic parameters were normal. Whole exome sequencing showed a hemizygous mutation (NM_003688;
c.764G > A [p.Arg255His] in CASK. Sanger sequencing confirmed that his parents harbored wildtype CASK (Fig. 2C). This mutation has been reported twice in the ClinVar pathogenicity data.

The patient could not control his head and had no social smile at 1.5 years. Improvements in seizures, constipation, and developmental progress were not noted. Severe growth failure and microcephaly (head circumference, $41 \mathrm{~cm},-3 \mathrm{SD}$ ) were observed. Repeat brain MRI revealed more severe imaging evolution than the original scans (Fig. 2B). HD, microcephaly with $\mathrm{PCH}$, and infantile spasms were diagnosed.

\section{Discussion}

$\mathrm{HD}$ is a neuro-gastrointestinal disorder caused by factors affecting the integrity of the gastrointestinal-neural network and smooth muscle function. A study found an actin G2 alpha gene variant in $64 \%$ of the $\mathrm{HD}$ cases, indicating that smooth muscle function may cause $\mathrm{HD} .{ }^{4} \mathrm{CASK}$, a multi-domain scaffolding protein, is expressed in most human organ systems, including the digestive and nervous systems. CASK protein 4.1 interacts with actin molecules and promotes the formation of actin and spectrin microfilaments. CASK acts as an adaptor protein, linking transmembrane proteins to the cytoskeleton. ${ }^{5,6}$ CASK aberrations cause central neurological disorders, including intellectual disability, microcephaly with $\mathrm{PCH}$, and developmental epileptic encephalopathy. ${ }^{7,8}$

A few studies have reported dysfunction of $C A S K$ with esophageal carcinoma and colitis., ${ }^{2,3}$ This patient presented with refractory 

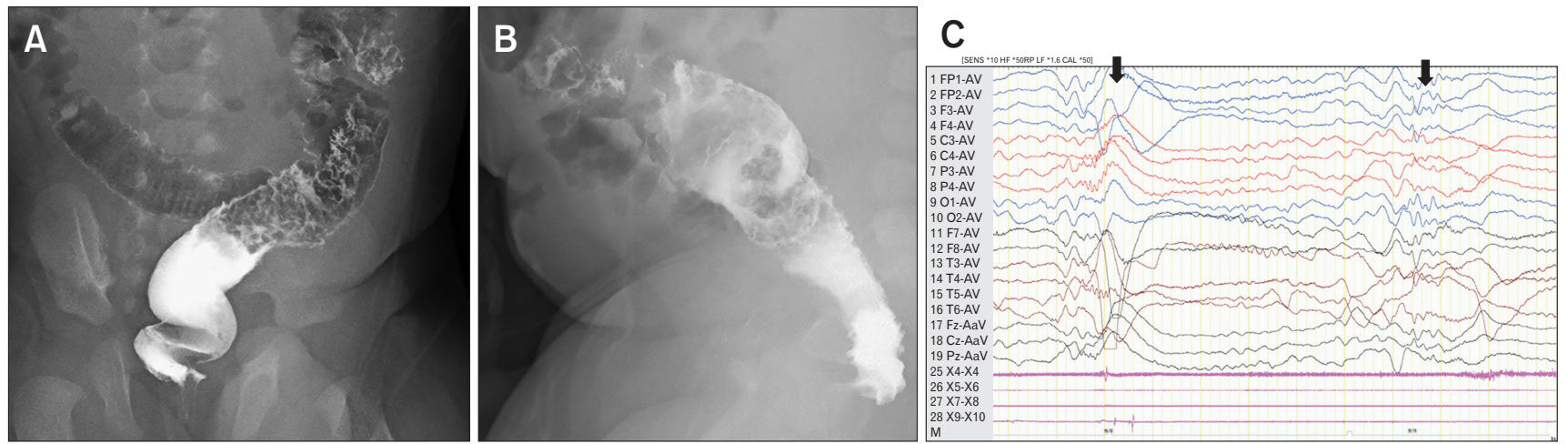

Figure 1. Abdominal radiographs showing significant bowel dilation with spasming of the distal colon (A, B). Electroencephalogram revealing a cluster of epileptic spasms (C).
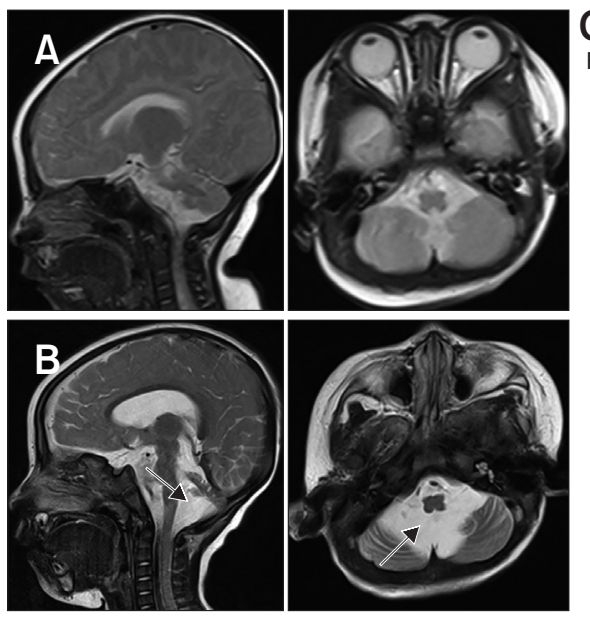

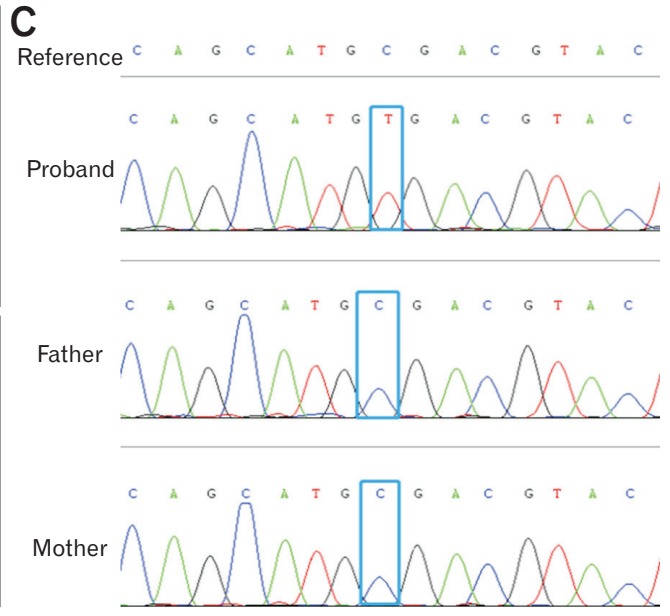

Figure 2. Brain MRI scans showing mild (A) and severe (B) pontocerebellar hypoplasia. Sequencing electropherograms illustrating the calcium/calmodulin-dependent serine protein kinase mutation (C). constipation with abdominal distension at onset, followed by neurological manifestations and displayed $\mathrm{HD}$ and $\mathrm{PCH}$ with $C A S K$ mutation. The CASK loss-of-function mutation led to the decreased expression of protein 4.1, which affected microfilament contraction and cell differentiation, leading to cytoskeletal abnormalities in the axons of the ganglion cells in the distal bowel and central nervous system; smooth muscle dysfunction, further contributed to these presentations. Our findings corroborate that CASK mutations can cause $\mathrm{HD}$ by affecting neuromodulation and smooth muscle function in the distal bowel.

We speculate that HD developed due to the mutation in CASK because of the following reasons: HD is related to ganglion cell defects or insufficient gastrointestinal motility, and dysfunction in cytoskeletal CASK affects enteric ganglion cell axon formation and intestinal smooth muscle function; the first disease manifestation in the patient was the digestive system symptoms, which persisted throughout the disease course, and typical neurological symptoms developed subsequently; $C A S K$ mutation was a pathogenic mutation.

This is the first report on HD and typical neurological presentations due to $C A S K$ mutations. Our preliminary study discusses the $C A S K$ phenotype and suggests that $C A S K$ mutations may underlie HD development, further providing expansion for the clinical and genetic spectrum of $\mathrm{HD}$ and the phenotype of CASK mutation.

\section{Maoqiang Tian, ${ }^{1 *}$ Hong Pan, ${ }^{2}$ Wenting Lei, ${ }^{1}$ Jing Chen, ${ }^{1}$ Juan Li, ${ }^{1}$ Changhui Lang, ${ }^{1}$ and Xiaomei Shu ${ }^{1}$ \\ Departments of ${ }^{1}$ Pediatrics and ${ }^{2}$ Cosmetic Skin Laser, Affiliated Hospital of Zunyi Medical University, Zunyi, China}

1. Tran VQ, Mahler T, Bontems P, et al. Interest of anorectal manometry during long-term follow-up of patients operated on for Hirschsprung's disease. J Neurogastroenterol Motil 2018;24:70-78.

2. Wang Q, Lu J, Yang C, et al. CASK and its target gene Reelin were coupregulated in human esophageal carcinoma. Cancer Lett 2002;179:71- 
77.

3. Carvajal AE, Vázquez-Carretero MD, García-Miranda P, Peral MJ, Calonge ML, Ilundain AA. Reelin expression is up-regulated in mice colon in response to acute colitis and provides resistance against colitis. Biochim Biophys Acta Mol Basis Dis 2017;1863:462-473.

4. Moore SW, Maluleke T, El Hosny AA. Is Hirschsprung disease a purely neurological condition? A study of the Actin G2 smooth muscle gene in Hirschsprung disease. J Pediatr Surg 2019;54:2028-2031.

5. Cohen AR, Wood DF, Marfatia SM, Walther Z, Chishti AH, Anderson JM. Human CASK/LIN-2 binds syndecan-2 and protein 4.1 and localizes to the basolateral membrane of epithelial cells. J Cell Biol 1998;142:129-138.

6. Hsueh YP. The role of the MAGUK protein CASK in neural development and synaptic function. Curr Med Chem 2006;13:1915-1927.

7. Takanashi J, Okamoto N, Yamamoto Y, et al. Clinical and radiological features of Japanese patients with a severe phenotype due to CASK mutations. Am J Med Genet A 2012;158A:3112-3118.
8. Nakajiri T, Kobayashi K, Okamoto N, et al. Late-onset epileptic spasms in a female patient with a CASK mutation. Brain Dev 2015;37:919-923.

Acknowledgements: Maoqiang Tian and Hong Pan contributed equally to this study. We would like to thank the patient and his parents for participating in this study. The parents of the patient have provided their written informed consent for publication.

Financial support: This study was supported by grants from the National Natural Science Foundation of China (Grant No. 31260286).

Conflicts of interest: None.

Author contributions: All authors participated in the diagnosis of the patient. Maoqiang Tian and Hong Pan collected clinical information and drafted the article; Jing Chen and Juan Li examined the gene sequencing results; Wenting Lei wrote the first draft; Changhui Lang studied the electroencephalogram; and Xiaomei Shu revised the article and gave substantial financial support. 